\title{
Der Finanzausgleich
}

\section{Wozu überflüssige Überlegungen anstellen?}

Die zweite Stufe der Föderalismusreform sollte sich auch mit dem Finanzausgleich befassen. Es ist aber nicht zu erwarten, dass dies geschieht. Zum einen fehlt, jetzt schon absehbar, die Zeit dazu. ${ }^{1}$ Zum anderen ist Anfang des Jahrhunderts der Finanzausgleich in seinem Kern auf eine ungewöhnlich lange Zeit hin, nämlich bis 2019 geregelt worden, eine seit Bestehen der Bundesrepublik einmalige Leistung.

Gleichwohl lohnen sich Überlegungen zum Finanzausgleich. Die Politik wird spätestens $^{2} 2015$ vor der Frage stehen, wie man die Nachfolge der nur bis 2019 geltenden Regeln über den Finanzausgleich organisiert. Sie wäre gut beraten, schon mit den Vorschlägen für den vermutlich auf die Schuldenfrage und Verwaltungsthemen beschränkten Entwurf für eine Verfassungsänderung das Verfahren und möglicherweise auch die Eckpunkte für die Fortsetzung der Finanzausgleichsregeln festzulegen.

Eine systematische Erfassung der mit dem Finanzausgleich verbundenen Probleme kann die auch dann noch hart genug werdenden Verhandlungen entlasten. Sie erlaubt, Scheinargumente $\mathrm{zu}$ erkennen und $\mathrm{zu}$ benennen, realistische Optionen aufzuzeigen und die Leistungsgrenzen der zunehmend in den Problemkomplex eingeführten wirtschaftswissenschaftlichen Denkfiguren zu bestimmen.

\section{Was ist der Finanzausgleich nach dem Grundgesetz?}

Auf den ersten Blick scheint es müßig, sich mit Begriff, Gestalt und Bedeutung des Finanzausgleichs nach dem Grundgesetz zu befassen, wenn man auch oder vor allem die Option einer Verfassungsänderung in den Blick nehmen will. Der Finanzausgleich stünde als Ganzer oder in der jetzigen Form, jedenfalls theoretisch, zur Disposition. Es wäre aber unprofessionell, wenn man sich nicht die strukturelle Einbindung des Fi-

1 Anders als die Bundesstaatskommission hat die »Gemeinsame Kommission zur Modernisierung der Bund-Länder-Finanzbeziehungen« eine nur vage Aufgabenstellung (BTDrucks. 16/3885); es lässt sich auch nicht sagen, dass ihr Zeitmanagement besonders vorteilhaft wäre. Schließlich gäbe es bei einer relevanten Änderung des Finanzausgleichs in der Höhe ausrechenbare Verlierer und Gewinner. Das ist keine gute Option für eine konzeptionell überzeugende Verfassungsänderung. Sie ist längst schon zu einer Verhandlungslösung degeneriert, bei der jede Verliererseite schon bei den Beratungen eine Vetoposition hat (BT-Drucks. 16/3885 sub 8).

2 Verhandlungen bei dieser Materie dauern erfahrungsgemäß Jahre. Die Politik braucht aber gerade in Finanzsachen Sicherheit. 2015 liegt nach normalem Verlauf in der Mitte einer Legislaturperiode. Anderthalb von Bundestagswahlen und ihren Vorwirren nicht gestörte Jahre sind mindestens notwendig. Ein späterer Beginn als 2015 kommt wegen der Bundestagswahl 2017 zu nahe an den Ablösungstermin 2019 heran. 
nanzausgleichs in das Finanzverfassungssystem des Grundgesetzes bewusst machte und damit die besondere Gestalt des deutschen Föderalismus.

Schon die Beantwortung der ersten Frage, was denn eigentlich »Finanzausgleich« meint, welche Regeln der Finanzverfassung also ihm zuzuordnen sind, ist schwieriger, als man annehmen würde. Umfasst er nur Finanzverschiebungen zwischen den Gebietskörperschaften Bund, Länder und Gemeinden, nachdem die Steuererträge als solche verteilt sind, oder kann auch die sogenannte Ertragshoheit, also der verfassungsrechtliche Anspruch auf einen Steuerertrag, schon Bestandteil des Finanzausgleichs sein? Diese Frage stellt sich, weil das Aufkommen der einzelnen Steuern, also die Steuererträge, von der Verfassung in unterschiedlicher Weise verteilt wird.

Dabei ist nicht so sehr entscheidend, dass die Verfassung die Erträge bestimmter Steuern ganz dem Bund (Bundessteuern: Art. 106 Abs. 1 GG), ganz den Ländern (Landessteuern: Art. 106 Abs. 2 GG) oder ganz den Gemeinden (Gemeindesteuern: Art. 106 Abs. $6 \mathrm{GG}^{3}$ ) zuweist und das Aufkommen der beiden weitaus größten Steuern dem Bund und der Ländergesamtheit und auch der Gesamtheit der Gemeinden nach Quoten zufließt ${ }^{4}$ (Gemeinschaftsteuern). Entscheidend ist vielmehr, dass bei den meisten Steuern das örtliche Aufkommen, also zum Beispiel das Aufkommen im Land, für die Zuweisung des Ertrages maßgebend ist, wie bei allen Landessteuern (Art. 106 Abs. 2 i. V. m. Art. 107 Abs. 1 Satz 1 GG). Bei zwei wichtigen Gemeinschaftsteuern, nämlich der Einkommen- und der Körperschaftsteuer, wird zwar eine Quote zwischen Bund und Ländergesamtheit von der Verfassung festgelegt, ${ }^{5}$ die Länderquote wird aber ebenfalls nicht nach Bedarf oder Größe auf die einzelnen Länder verteilt, sondern wie bei den Landessteuern nach dem örtlichen Aufkommen (Art. 107 Abs. 1 Satz 1 GG).

Das Aufkommen im Land ist also für die Landessteuern und die Landesanteile an den oben genannten Gemeinschaftsteuern maßgebend. Ob man daraus auf die Vorstellung der Verfassung zu schließen hat, dass das Land die entsprechenden Steuererträge in einem substanziellen Sinne »erwirtschaftet $\aleph^{6}$ hat, ist später zu klären. Hier genügt die Feststellung, dass die Ertragsverteilung bei diesen Steuern jedenfalls nicht speziell auf den Bedarf der Gebietskörperschaften abstellt und sich daher auch darüber ausschweigt, ob zwischen einem selbst verursachten oder von außen erzwungenen Bedarf zu differenzieren ist. Sie hat insofern keine finanzausgleichsrechtliche Dimension. Das

3 Teilweise können sie von den Ländern auch den Gemeindeverbänden zugewiesen werden (Art. 106 Abs. 1 Satz 1 GG), was nicht geschehen ist. Das ausgeübte Umlagerecht (Art. 106 Abs. 6 Satz 4 GG) lässt die Ertragshoheit an sich unberührt.

4 Oder zu quotieren dem Gesetzgeber aufgibt, wie bei dem Anteil der Gemeinden an der Einkommensteuer (Art. 106 Abs. 3 GG). Bei der Körperschaftsteuer gibt es nur eine Aufteilung zwischen dem Bund und der Ländergesamtheit.

5 Es handelt sich in beiden Fällen um eine hälftige Aufteilung (Art. 106 Abs. 3 Satz 1 GG). Vom Aufkommen der Einkommensteuer ist aber vorweg eine durch einfaches Gesetz festgelegte Quote für die Gemeindegesamtheit abzuziehen (Art. 106 Abs. 3 Satz 1 Hs. 2 GG); sie beträgt schon sehr lange Zeit $15 \%$.

6 Wie BVerfGE 72, 330, 384 oder 116, 327, 379 es formulieren. 
Charakteristikum des Finanzausgleichs nach dem Grundgesetz besteht nämlich in dem Versuch, einen durch die geschilderte Ertragsverteilung nicht gedeckten Bedarf möglichst zu befriedigen. ${ }^{7}$

Soweit also die Steuerertragsverteilung nicht darauf zielt, gehört sie nicht zum Finanzausgleich. Das gilt für die verfassungskräftige Zuordnung der Erträge bei den Bundessteuern, bei den Landessteuern ${ }^{8}$ wie bei den Gemeindesteuern sowie für die Länderanteile an den beiden oben genannten Gemeinschaftsteuern.

\section{Der primäre Finanzausgleich}

An zwei Stellen verfolgt schon die Zuordnung der Erträge von Steuern in der Verfassung aber einen speziell auf den Bedarf abstellenden Zweck. Der vom Volumen bedeutsamste Fall ist die Verteilung des Umsatzsteueraufkommens, also des Ertrages der zweiten sehr ertragreichen Steuer. Hier geht es um zwei scharf voneinander zu trennende Komplexe, zum einen um die Verteilung des Aufkommens zwischen dem Bund auf der einen und der Ländergesamtheit auf der anderen Seite. Zum anderen geht es um die Verteilung des so ermittelten »Länderanteils« auf die einzelnen Länder. Die politische Bedeutung dieses gestuften Verteilungssystems lässt sich ironischer Weise an der Unsinnigkeit einiger Details der Regelung ebenso erkennen wie an der Tatsache, dass die Staatspraxis einen Verfassungsbefehl und Auflagen des Bundesverfassungsgerichts schlicht nicht befolgt.

Was zunächst die Verteilung des Ertrages zwischen dem Bund und der Ländergesamtheit angeht, so enthält Art. 106 Abs. 3 Satz 4 GG in den Nr. 1 und 2 dieser Vorschrift nämlich Anforderungen, die in diesem Stadium des Verfahrens nicht erfüllbar sind. Streng genommen hat schon der erste, an sich sehr vernünftig klingende Verteilungsmaßstab keinen rechten Sinn, dass nämlich »der Bund und die Länder« gleichmäßig Anspruch auf Deckung der notwendigen Ausgaben haben. Bei dieser Oberteilung sind nämlich gar nicht »die Länder«, sondern ist die Ländergesamtheit der Bezugspunkt. Diese kann als Gesamtheit keine »notwendigen Ausgaben« haben. Den notwendigen Ausgaben des einzelnen Landes wiederum kann gar nicht Rechnung getragen werden, weil die Verfassung den Länderanteil auf die einzelnen Länder zu mindestens $75 \%$ nach der Kopfzahl verteilt und zu höchstens 25\% nach der Finanzschwä-

7 Freilich wird der Begriff »Finanzausgleich« vor allem von den Finanzwissenschaften auch in einem sehr weiten Sinne verstanden als der Inbegriff aller wesentlichen Finanzrechte in einem gegliederten Staatswesen. Als solcher ist er aber wenig aussagefähig. Und auch die unterschiedslose Einbeziehung aller auf die Einnahmeerzielung und Einnahmenverteilung zielenden Regelungen kürzen den Begriff »Ausgleich« um ein wesentliches Element. Für die vorliegende Untersuchung ist aber die Differenzierung zwischen der Ertragsverteilung, die bedarfsunabhängig erfolgt, und der dieses Ergebnis ausgleichenden Ertragsverteilung entscheidend. Der Begriff wird hier also in einem engeren Sinne benutzt und schließt so an den von der Verfassung in Art. 107 Abs. 2 Satz 1 GG selbst verwendeten Sinn an.

8 Einschließlich der Gewerbesteuerumlage zugunsten der Länder, die Art. 106 Abs. 6 Satz 4 GG erlaubt und die $\S 6$ Gemeindefinanzreformgesetz außerordentlich kompliziert regelt, indem er die Gemeinden auch in die Mitfinanzierung der Kosten der deutschen Einheit einbezieht. Nach § 3 FAG ist für die Länder dabei das örtliche Aufkommen maßgebend. 
che (Art. 107 Abs. 1 Satz 4), nicht aber nach dem notwendigen Bedarf eines Landes. Eine Erhöhung des Länderanteils würde allen Ländern unabhängig von ihrem Bedarf zu Gute kommen. Zum zweiten ist die nach Nr. 2 zu vermeidende »Überbelastung der Steuerpflichtigen« in dieser Phase der Verteilung eines Aufkommens gar nicht mehr zu erfüllen, die Anforderung müsste sich an den Steuergesetzgeber richten. ${ }^{9}$ Schließlich lässt sich die ebenfalls in Nr. 2 aufgestellte Anforderung, »die Einheitlichkeit der Lebensverhältnisse im Bundesgebiet zu wahren«, wegen der von derselben Verfassung vorgeschriebenen Pro-Kopf-Verteilung auf die einzelnen Länder auch nicht erfüllen.

Bei so viel verfassungsrechtlicher Lyrik verwundert es nicht, dass handfestere Anforderungen wie die Ermittlung des Umfangs der Ausgaben anhand einer mehrjährigen Finanzplanung von der Staatspraxis schlicht ignoriert werden ${ }^{10}$ und dass die Politik sich keineswegs an die Forderung des Bundesverfassungsgerichts gehalten hat, im dem von diesem geforderten Maßstäbegeset $\mathrm{z}^{11}$ Konkretisierungen und Kriterien für die Umsatzsteuerverteilung festzulegen.

Die Crux des Verteilungsbefehls in Art. 106 Abs. 3 Satz 4 GG ist, dass die Notwendigkeit von Ausgaben sich im Kern nicht bestimmen lässt, weil sie sich aus politischen Setzungen ergibt, selbst wenn sie sich in gesetzlich fixierten Ansprüchen Dritter äußern. Hochrangige Fachleute beider Seiten sprechen von einer nicht auflösbaren Differenz zwischen Bund und Ländern von 7 bis 8 Milliarden. ${ }^{12}$ Die neuere Staatspraxis geht dazu über, den status quo als Maßstab zu nehmen und für zwischenzeitliche Änderungen der Verpflichtungen Zu- und Abschläge auszuwerfen, was den § 1 FAG, der die Verteilung des Umsatzsteuerertrages zwischen Bund und Ländern regelt, nachgerade unlesbar gemacht hat. ${ }^{13}$ Am Einfachsten ist noch die Aussage, dass die Gemeinden insgesamt 2,2\% des Umsatzsteuerertrages erhalten, aber eben nicht von 100\% dieses Ertrages, sondern

9 Aus dieser doch höchst speziellen Stelle, an der die Anforderung aufgestellt ist, gleich auf eine allgemeines Verfassungsprinzip zu schließen, wie das in der ersten großen oder jedenfalls langen Bearbeitung des Art. 106 geschehen ist (Vogel/Walter, Bonner Kommentar, Art. 106 (1972, Rn. 54, 181), dürfte Kommentierung mit Verfassungsgebung verwechseln.

10 Ohne Erfolg gerügt von BVerfGE 101, 158, 227.

11 Die Einforderung eines solchen Gesetzes ist freilich eine Erfindung des Bundesverfassungsgerichts und zwar keine besonders überzeugende (BVerfGE 101, 158, 214 ff.). Die Umsetzung der Finanzverfassung soll nach ständiger Rechtsprechung allen Gliedern des Bundes eine hinreichende Finanzausstattung zur Erfüllung ihrer Aufgaben geben. Man kann dann aber dem Gesetzgeber nicht zumuten, ihn selbst bindende Verteilungsregeln in Unkenntnis ihrer Auswirkungen zu machen. Maßstäbegesetz und Finanzausgleichsgesetz wurden denn auch, entgegen der Vorgabe des Gerichts, im Zusammenhang ausgearbeitet. Keine ernsthaften Gedanken hat sich das Gericht über die Stellung des Gesetzes in der Ordnung der Rechtsquellen gemacht. Das Gesetz selbst beschränkt sich im Wesentlichen auf die Wiedergabe solcher Einzelaussagen aus Urteilen, die für die Politik akzeptabel schienen.

12 So übereinstimmend der Ministerpräsident Koch und der ehemalige Erste Bürgermeister und nunmehr Bundestagsabgeordnete Runde bei den Beratungen der Bundesstaatskommission zur Föderalismusreform 2006.

13 Außerdem kümmert sich diese Praxis, die 2001 auf die Spitze getrieben worden ist, nicht um die Einwände, die das Gericht in BVerfGE 101, 158, 214 ff. (228) formuliert hat. 
von einem - jedenfalls ab dem Jahre 2009 - um zehn Prozentpunkte zu Gunsten des Bundes gekürzten Umsatzsteuerertrag. Das ist aber, wie gesagt, noch die einfachste Rechnung. Jedenfalls scheint man bei der Festlegung der Anteile am Umsatzsteuerertrag nicht die jeweilige Gesamtbelastung im Auge zu haben, die sich ja jenseits der gesetzlich verursachten Änderungen durchaus nicht unerheblich ändern kann, sondern sich auf Zusatzbe- und Entlastungen zu konzentrieren. Mit Eurobeträgen auch diesseits der Millionenbeträge wird eine Scheingenauigkeit vorgespielt, ${ }^{14}$ die angesichts der Ungenauigkeit von 7 bis 8 Milliarden Euro bei der Ausgangsrechnung eher kurios wirkt.

Bei dem zweiten Akt der Verteilung, nämlich der Verteilung des Länderanteils auf die einzelnen Länder, wird die Umsatzsteuer nicht nach dem örtlichen Aufkommen verteilt, sondern nach der Einwohnerzahl (Art. 107 Abs. 1 Satz 4 GG). Mit dem zwingenden Bezug auf die vorhandenen »Köpfe« ist für die Verteilung ein zwar höchst allgemeines, gelegentlich wird auch gesagt »abstraktes«, aber eindeutiges Bedarfsargument zum tragenden Verteilungsmaßstab geworden. ${ }^{15}$ Darüber hinaus können für ein Viertel des gesamten Länderanteils an der Umsatzsteuer nach derselben Vorschrift Ergänzungsanteile für die Länder vorgesehen werden, »deren Einnahmen aus den Landessteuern und der Einkommensteuer und der Körperschaftsteuer je Einwohner unter dem Durchschnitt der Länder liegen«. Diese Option, die bisher immer genutzt worden ist, hat eine zusätzliche Ausgleichsfunktion.

Den anderen Fall einer bedarfsorientierten Ertragsverteilung gibt es bei der Detailverteilung des den Gemeinden insgesamt zustehenden 15\%-igen Anteils an der Einkommensteuer ( $\$ 1$ Gemeindefinanzreformgesetz). ${ }^{16}$ Nach Art. 106 Abs. 5 Satz 1 GG ist der Gemeindeanteil nämlich nicht nach dem örtlichen Aufkommen in der Gemeinde, ${ }^{17}$ sondern nur »auf der Grundlage der Einkommensteuerleistung in der Ge-

14 So stehen dem Bund nach Abzug der oben schon genannten zehn Prozentpunkte zu seinen Gunsten und nach dem Abzug der 2,2 \% für die Gemeinden von den verbleibenden 90\%, also von den verbleibenden 88,02\% des Gesamtertrages der Umsatzsteuer im Jahre 2009 49,5\% zu, nicht ohne dass ihm davon 2262712000 Euro abgezogen werden. Von einer von der Verfassung eigentlich gewollten und bisher immer betriebenen quotalen Aufteilung des Umsatzsteuerertrages ist nur noch ein Torso übrig geblieben. Vom früheren »Steuerstreit« ist man zum Stellungskrieg übergegangen.

15 Finanzwissenschaftler mögen dies damit begründen, dass das örtliche Aufkommen nicht oder nicht immer der wirtschaftlichen Wirkung der Steuer entspreche. Auch in BVerfGE 72, 333, 384 wird eine Art »Rechtfertigung « in diesem Argument gesehen. Um eine Rechtfertigung bemüht sich auch Stefan Korioth, Der Finanzausgleich zwischen Bund und Ländern, 1997, 526 f. Einer Rechtfertigung bedarf der Staat möglicherweise für die Steuerhebung als solche, warum er sie aber für die Verteilung eines Teils des Steueraufkommens zwischen den Ländern benötigte, bleibt dunkel. Das schlichteste Argument ist, dass bei einer Verteilung nach dem örtlichen Aufkommen der sehr viel prekärere Länderfinanzausgleich, also der horizontale Finanzausgleich, über die Maßen hätte belastet werden müssen. Die Hessen mit ihren hohen Finanzumsätzen in Frankfurt würden sich allerdings freuen.

16 Von 2001 (BGBl. I S. 482), zuletzt geändert durch Gesetz vom 14. 8. 2007 (BGBl. 2007 I S. 1912).

17 Der allen Gemeinden eines Landes vorbehaltene Anteil von 15\% des Aufkommens bemisst sich nach dem örtlichen Aufkommen im jeweiligen Land. Art. 106 Abs. 5 Satz 1 GG verfügt das zwar nicht unmittelbar, es ist aber bei systematischer Betrachtung aus dem Kontext zu erschließen. 
meinde« weiterzureichen. Praktisch führt das zugunsten der schwächeren Gemeinden bei der Bemessung des Anspruchs für $»$ reiche Gemeinden $\aleph^{18}$ zu einer nicht unerheblichen Kupierung des Aufkommens einkommensstarker Bürger, also des örtlichen Aufkommens, indem die Einkommensteuerleistung einer Person ab einer ziemlich niedrigen Grenze der Gemeinde nicht zugerechnet wird. § 3 Abs. 1 Gemeindefinanzreformgesetz setzt diese Grenze grundsätzlich bei 30.000 Euro zu versteuernder Einkommensbeträge fest. ${ }^{19}$

Man kann diese Ausgleichselemente schon bei der Ertragsverteilung der Steuern als den primären Finanzausgleich ${ }^{20}$ bezeichnen. Eigenartiger Weise ist er, was die Ertragszuweisung an die Länder angeht, in der politischen Debatte anders als der anschließend zu skizzierende sekundäre Finanzausgleich nie im Visier der starken Länder gewesen. Er wird von ihnen als selbstverständlich hingenommen, obwohl es sich bei der Umsatzsteuer um erhebliche Beträge handelt. Ein Grund könnte sein, dass die nicht unerheblichen Finanzmassen, um die es geht, nämlich der Umsatzsteueranteil der Länder, anders als die Mittel des sekundären Finanzausgleichs, gar nicht erst in ihren Kassen landen, von ihnen also auch nicht herausgegeben werden müssen. ${ }^{21}$ Man mag dabei an die Einkommensteuer denken, die, soweit sie vom Arbeitsentgelt abgezogen wird, weniger zu schmerzen pflegt als die nach Veranlagung abzuführende Steuer, ${ }^{22}$ und zwar durchaus unabhängig von der jeweiligen Größenordnung. Bei der ausgleichenden Ertragszuweisung des Einkommensteueranteils an die Gemeinden dagegen regt sich kein Widerstand der Länder, weil sie gewohnt sind, über den kommunalen Finanzausgleich, für den Art. 106 Abs. 7 GG die Grundlage gibt, unterschiedliche Bedarfe ihrer Gemeinden zu bedienen.

\section{Der sekundäre Finanzausgleich}

Wie so manche Regel in der Finanzverfassung ist die dem sekundären Finanzausgleich zugrunde liegende Vorschrift weit davon entfernt, ein Musterstück der Gesetzgebungskunst zu sein. Sie steht in Art. 107 Abs. 2 Satz 1 GG und lautet: »Durch das Gesetz ist sicherzustellen, dass die unterschiedliche Finanzkraft der Länder angemes-

18 Also Gemeinden mit einkommensstarken Bürgern.

19 Keinen wesentlichen Bedarfsgedanken enthält die in Art. 106 Abs. 5a GG angeordnete Verteilung des Anteils der Gemeinden am Umsatzsteuerertrag, die »auf der Grundlage eines orts- und wirtschaftsbezogenen Schlüssels « zu erfolgen hat und nicht nach dem örtlichen Aufkommen. Hier geht es um Kompensationen für eine aufkommensmindernde Änderung des Gewerbesteuerrechts. Die Umsetzung durch $\S \S 5$ bis $5 \mathrm{~d}$ des Gemeindefinanzreformgesetzes splittet zunächst die Verteilungsmasse zwischen den neuen Bundesländern einschließlich Ostberlins und den alten im Verhältnis $15 \mathrm{zu} 85$ auf, worin wohl Ausgleichselemente enthalten sind, und stellt dann einerseits auf Gewerbesteueraufkommen und die Zahl der versicherungspflichtigen Beschäftigten und andererseits auf Gewerbesteuermessbeträge ab.

20 Der im Übrigen, wie in Fn. 6 erläutert, unterschiedslos für alle unmittelbaren Zuweisungen von Steuererträgen benutzt wird.

21 Steuergläubiger ist nach Art. 108 Abs. 1 GG der Bund. Steuerertragshoheit und Steuergläubigerschaft fallen also beim Länderanteil an der Umsatzsteuer auseinander.

22 Auf diese Idee hat mich Joachim Wieland gebracht. 
sen ausgeglichen wird; hierbei sind die Finanzkraft und der Finanzbedarf der Gemeinden (Gemeindeverbände) zu berücksichtigen.« Einzig vernünftig wäre aber der Beginn mit »Durch Gesetz ist sicherzustellen«. Denn »das « Gesetz verweist auf ein vorher genanntes Gesetz. Das aber ist das in Art. 107 Abs. 1 Satz 4 Hs. 2 GG genannte Gesetz, das ermöglichen soll, ein Viertel des Länderanteils an der Umsatzsteuer nach genuin finanzausgleichsrechtlichen Gesichtspunkten zu verteilen. Dieses Gesetz kann aber wegen des von ihm zu regelnden Volumens und der Bedingungen, denen es unterworfen ist, die von ihm verlangte Funktion gar nicht erfüllen; vor allem aber ist es nach Art. 107 Abs. 1 Satz 4 GG kein notwendiges Gesetz. Erst mit den Formulierungen des Art. 107 Abs. 1 Satz 2 GG wird klar, dass der Inhalt des hier geforderten Gesetzes mit dem in Art. 107 Abs. 1 Satz 4 Hs. 2 GG geforderten unmittelbar nichts zu tun hat, sondern eine neue Stufe des Finanzausgleichs eröffnet.

Dieser sekundäre Finanzausgleich, der die Verschiebung von Finanzmassen unabhängig von Steuererträgen ${ }^{23}$ vorzunehmen befiehlt, tritt in zwei Formen auf. Einmal geht es um den ausschließlich zwischen den Ländern vorzunehmenden, aber vom Bund zu organisierenden, ${ }^{24}$ horizontalen Finanzausgleich, der auch Länderfinanzausgleich genannt wird, und zum anderen geht es um einen vom Bund zu den Ländern gehenden Finanzausgleich, der auch vertikaler Finanzausgleich genannt wird. Er wird vom Bund in Form von Bundesergänzungszuweisungen realisiert. Der horizontale Finanzausgleich ist obligatorisch, der vertikale zwar nach der Verfassung nicht, wohl aber in einem politischen Sinne, nämlich nach den Gepflogenheiten des Aushandelns, die das Feld des Finanzausgleichs in besonderem Maße bestimmen. Es liegt auf der Hand, dass die finanzstarken Länder nichts gegen Bundesergänzungszuweisungen einzuwenden haben, wohl aber, wenn sie im horizontalen Finanzausgleich zur Kasse gebeten werden. So verwundert es nicht, dass bei dem bis 2019 geregelten Finanzausgleich die Bundesergänzungszuweisungen in Form der »Sonderbedarfs-Bundesergänzungszuweisungen « (§ 11 Abs. 3 FAG) jedenfalls in den ersten Jahren exorbitant hoch sind. Sie beginnen mit zehneinhalb Milliarden im Jahre 2005 - und sind damit nicht unerheblich höher als das Volumen des horizontalen Finanzausgleichs zwischen den Ländern - und enden mit gut zwei Milliarden im Jahre 2019. ${ }^{25}$ Die Einigung zwischen den Ländern zum horizontalen Finanzausgleich wird immer zu Lasten des Bundes herzustellen versucht.

23 Die Steuererträge spielen nur bei der Bemessung der Ausgleichsleistungen, dann aber eine wichtige Rolle. Rechtlich bestehen die Ansprüche gegen die verpflichteten Gebietskörperschaften, aus welchen Einnahmen diese sie erfüllen, ist ihnen überlassen.

24 Nämlich durch Zustimmungsgesetz: Art. 107 Abs. 2 i. V. m. Art. 107 Abs. 1 Satz 4 GG.

25 Siehe $\S 11$ Abs. 3 FAG. Hinzu kommen noch »Sonderbedarfs-Bundesergänzungszuweisungen«, die insgesamt 1,5 Milliarden Euro ausmachen, auf Notwendigkeit und Höhe aber nach einer festgelegten Zeit zu überprüfen sind. 


\section{Der horizontale Finanzausgleich}

Beim horizontalen Finanzausgleich als wichtigem Bestandteil des sekundären Finanzausgleichs geht es um Gelder, welche die finanzstarken Länder unabhängig von der vorangegangenen Steuerertragsverteilung zu Gunsten der finanzschwachen Länder zu geben verpflichtet sind. Er steht in der immer wieder aufflammenden Kritik finanzstarker Länder, wobei sie sich, wenn es zu passen scheint, der Hilfe der Wirtschaftswissenschaften zu bedienen pflegen. Es geht um den Vorwurf unzulässiger Nivellierung, dass sich »politische Arbeit« nicht, wohl aber eine Rikscha-Mentalität lohne, es keinen Anreiz gebe, ein hohes Steueraufkommen zu erwirtschaften und dass die finanzstarken Länder ohne diese Ausgleichsverpflichtung sich nicht verschuldet hätten. ${ }^{26}$ Im Zentrum der Auseinandersetzungen steht also die Verschiebung von Finanzmassen zwischen den Ländern, und zwar auf der Grundlage eines zustimmungspflichtigen Bundesgesetzes.

Art. 107 Abs. 2 Satz 1 GG formuliert in seinem ersten Halbsatz: »Durch das Gesetz ist sicherzustellen, dass die unterschiedliche Finanzkraft der Länder angemessen ausgeglichen wird. $\ll^{27}$ Erstaunlich ist an der Formulierung von Satz 1, dass der Finanzausgleich nicht ausdrücklich die Relation von Finanzkraft und Finanzbedarf eines Landes zum Maßstab macht, sondern allein seine Finanzkraft, das heißt, seine Einnahmepotenz. Und auch die Formulierung des zweiten Halbsatzes der Vorschrift, »hierbei sind die Finanzkraft und der Finanzbedarf der Gemeinden (Gemeindeverbände) zu berücksichtigen«, ist auf den ersten Blick nicht hilfreich, weil das argumentum e contrario nahe liegt, dass bei den Ländern der Finanzbedarf eben nicht zu berücksichtigen sei. Nimmt man hinzu, dass bei der Festlegung der Ertragsquoten der Umsatzsteuer zwischen Bund und Ländergesamtheit nach Art. 106 Abs. 3 Satz 3 und 4 GG »die notwendigen Ausgaben «, also der Bedarf, eine dominante Rolle spielen soll, könnte man sich in dem Gedanken bestätigt fühlen, der Bedarf der einzelnen Länder solle nur hier und nicht beim Finanzausgleich eine Rolle spielen.

Der Eindruck täuscht jedoch. Schon das letzte Argument ist nicht valide, weil Art. 106 Abs. 3 Satz 3 und 4 GG nur eine Rechnung zwischen Bund und Ländergesamtheit kennt, also Bedarfe einzelner Länder dabei nicht zu berücksichtigen sind und auch nicht berücksichtigt werden können. Die Verteilung der Länderquote auf die einzelnen Länder richtet sich nämlich nicht an speziellen Bedarfen aus, sondern erfolgt von Verfassungs wegen grundsätzlich nach Köpfen, wie gezeigt worden ist. Aber auch der Verweis auf die genannte Kommunalregel des Art. 107 Abs. 2 Satz 1 Hs. 2 GG mit dem ausdrücklichen Hinweis auf den Bedarf führt in die Irre.

26 Das haben der hessische Ministerpräsident und sein Finanzminister zur Rechtfertigung ihrer Verschuldenspolitik mehrmals erklärt, dürfte aber auch in den Regierungen anderer finanzstarker Länder gedacht werden. In seinem Beitrag »Ein Generationenvertrag über den Weg aus der Schuldenfalle« (FAZ v. 6. April 2008) hat der hessische Ministerpräsident Koch die vorgeschlagenen Zinshilfen für Länder, die ihren Zinsdienst bei dem vorgesehenen Schuldenfonds nicht tragen können, nicht als Ersatz für den Länderfinanzausgleich bezeichnet, aber gemeint, »sie würden dazu beitragen, die Diskussionen um einen bedarfsorientierten Finanzausgleich zu beenden, was zu den zentralen Forderungen der Zahlerländer« gehöre. Ein »Generationenvertrag, in dem die nachfolgende Generation zur Abzahlung der von uns gemachten Schulden gegen das Versprechen sich verpflichten soll, dass wir keine zusätzlichen mehr machen werden, dürfte von der belasteten Generation kaum geschlossen werden.«

27 Dass es nur um Finanzverschiebungen zwischen Ländern geht, ergibt erst Satz 2 der Vorschrift, der von ausgleichspflichtigen und ausgleichberechtigten Ländern spricht. 
Diese zunächst überraschend erscheinende Regel erweist sich als notwendig, weil ein Finanzausgleich, auch nur auf das Land bezogen, ohne Rückgriff auf ein Bedarfskriterium nicht sinnvoll organisiert werden kann. Ein Ausgleich von Mitteln nur nach der Höhe der Mittel und ohne einen anderen Maßstab ergäbe keinen Sinn. Da die Länder für die Finanzausstattung der Kommunen zuständig sind und die Verantwortung tragen, soweit nicht das Grundgesetz den Kommunen Ertragsanteile zuweist, bedurfte es einer Anordnung, dass deren Finanzbedarf, und zwar soweit er nicht als Landesbedarf formuliert werden kann, in Rechnung zu stellen ist.

Die Notwendigkeit des Einbezuges eines Bedarfs bei der Festlegung der Finanzkraft eines jeden Landes ergibt sich daraus, dass die Finanzkraft, da sie eine Vergleichsgröße für einen Ausgleich zwischen den Länder abgeben soll, sinnvoll nicht ohne eine Relation zum Bedarf festgestellt werden kann. Sonst müssten große Länder, ob sie finanzstark oder finanzschwach sind, immer an kleine Länder wie das finanzstarke Hamburg zahlen, nur dank ihres größeren Etats. Es lag daher nahe, das Kriterium, was schon bei der Verteilung des Umsatzsteuerertrages maßgebend ist, nämlich die Kopfzahl, maßgebend sein zu lassen. Sie nennt $\S 8$ Abs. 1 MaßstG »ein abstraktes Bedarfskriterium«, das notwendig sei, »um die Finanzkraft der Länder vergleichbar zu machen«. Was daran »abstrakt« sein soll, bleibt etwas dunkel. In Wirklichkeit handelt es sich um ein sehr allgemeines, aber auch sehr grundlegendes, ja fundamentales Bedarfskriterium. ${ }^{28}$ Es hat den weiteren großen Vorteil, dass es von einer gewillkürten, allein politischen Zielen dienenden Vergrößerung des Bedarfs unabhängig ist. Es behandelt alle Länder gleich. Ob sie ihre Krippenplätze oder Schulen besser ausstatten, ihre Polizisten besser oder schlechter bezahlen, ist ihre politische Entscheidung, hat aber keinen Einfluss auf Ansprüche aus dem Finanzausgleich. Erst Recht spielt die Höhe der Pro-Kopf-Verschuldung keine Rolle. Ein horizontaler Finanzausgleich zwischen den Ländern wäre nicht sinnvoll, wenn es jedes Land in der Hand hätte, durch sein politisches Verhalten andere Länder zur Kasse zu bitten. Das durch die Kopfzahl ausgedrückte Bedarfselement ist also nachgerade ideal. Es erfasst einen Grundbedarf, der in allen Ländern einigermaßen gleichmäßig auftritt. ${ }^{29}$

28 Der Begriff ist offenbar aus BVerfGE 86, 148, 239 übernommen. Abstrakt meint danach »jedweder besonderen, aus spezifischen Situationen sowie eigenen Prioritäts- und Dringlichkeitsentscheidungen der Länder herrührenden Ausgabenlast vorgelagert«.

29 Es ist daher konsequent, wenn der Gesetzgeber in den Fällen, in denen das nicht der Fall ist, differenziert. Traditionell geschieht das durch »Veredelung der Köpfe«. So werden nach § 9 Abs. 2 und 3 FAG in den Stadtstaaten bei der Ermittlung des Bedarfs zur Anrechnung auf alle Steuererträge die Köpfe zu 135\% gezählt (schon in BVerfGE 72, 300, 401 akzeptiert, in BVerfGE 101, 158, 230 trotz heftiger Angriffe (dort S. 196) ohne nähere Begründung toleriert, in BVerfGE 86, 148, 223 ff. und in BVerfGE 101, 158, 230 ff. für eine Staffelung nach Gemeindegrößen innerhalb der Flächenländer in Zweifel gezogen, was 2001 zur Aufgabe führte) und die Köpfe nach der Anregung in BVerfGE 101, 158, 230 f. bei drei dünn besiedelten ostdeutschen Flächenländern zwischen 102\% und 105\% zur Anrechnung auf die Gemeindeeinnahmen ( 9 Abs. 3 FAG) angesetzt. Sonderbedarfe wie Hafenlasten, die ursprünglich - eher unverständlich - »aus Tradition « vom Gericht toleriert wurden (BVerfGE 72, 330, 400; 86, 148, 222f.), sind seit der Kritik in BVerfGE 101, 158, 229 aus der Berechnung ausgeschieden und werden über Art. 104b a. F. GG spätestens bis Ende 2019 vom Bund mitfinanziert. 
Dieses Bedarfskriterium umfasst den normalen kommunalen Bedarf im Land mit, weil die Landesbevölkerung mit der gesamten Kommunalbevölkerung im Land identisch ist. $§ 7$ Abs. 1 Satz 2 MaßstG stellt diese Rechtsfolge ausdrücklich fest. Daher meint die ausdrücklich angeordnete Anrechnung des kommunalen Finanzbedarfs nur spezielle kommunale Bedarfe ${ }^{30}$ und ergibt so erst einen Sinn.

Drei Fragen, die auch zu erheblichen Streitigkeiten vor dem Bundesverfassungsgericht geführt haben, beantwortet die Verfassung nicht: Was zählt zur Finanzkraft eines Landes, sind für die Festlegung des Bedarfs auch andere Maßstäbe als die Kopfzahl zulässig und vor allem, welche Nivellierungswirkung ist erlaubt. Welche Einnahmen der Länder anzurechnen sind, ist im Großen und Ganzen sinnvoll gelöst. Es gilt der Grundsatz, alle Einnahmen zu berücksichtigen, nicht notwendig nur Steuereinnahmen. ${ }^{31}$ Die Finanzkraft der Länder ist keine beliebige Größe, die durch Verhandlungen $\mathrm{zu}$ bestimmen wäre. Bagatellprobleme sind aber ebenso ausgeschieden wie alle im Verhältnis zur Einwohnerzahl gleichmäßig anfallenden Einnahmen, weil sie für den Ausgleich unerheblich sind, und solche Einnahmen, »bei denen der Aufwand für die Ermittlung der auszugleichenden Einnahmen zur möglichen Ausgleichswirkung außer Verhältnis steht«, wie $§ 7$ Abs.1 Maßstäbegesetz (MaßstG) in Anschluss an BVerfGE 72, 330, 400 formuliert.

Da die Steuereinnahmen der Länder auf Bundesgesetzen beruhen, fallen sie gleichmäßig an. Es ist den finanzstarken Ländern also nicht möglich, sich durch Steuersenkungen in der Hoffnung »ärmer « zu machen, dies über Ersparnisse im Länderfinanzausgleich kompensieren zu können. ${ }^{32}$ Bei manchen Äußerungen zum »Wettbewerbsföderalismus « hat man den Eindruck, dies wäre die ideale Form des Wettbewerbs, nämlich Wettbewerb durch weniger Steuern. Angesichts der hoffnungslos überschuldeten öffentlichen Hand ist das freilich eine frivole Idee. Das durch die Föderalismusreform 2006 den Ländern zugestandene Recht, den Steuersatz bei der durch Bundesgesetz geregelten Grunderwerbssteuer selbst festzulegen, ${ }^{33}$ hat zu der Befürchtung geführt, finanzstarke wie finanzschwache Länder könnten versucht sein, sich dieses Mechanismus zu bedienen. Daher wird für die Berechnung der Einnahmen eines jeden Landes aus dieser Steuer eine normative Annahme getroffen, die eine

30 So auch BVerfGE 86, 148, 223.

31 Neben den Einnahmen aus den eigenen Steuern und den Anteilen an den Gemeinschaftsteuern sind ausdrücklich noch die Einnahmen aus der Förderabgabe nach $\S 31$ BBergG aufgeführt, die mittlerweile ihre finanzielle Bedeutung verloren haben und erst nach einem Prozess vor dem Bundesverfassungsgericht aufgenommen wurden (BVerfGE 72, 330, 410 ff.), weil damals das »Nehmer«-Land Niedersachsen an die 2 Milliarden DM solcher Einnahmen hatte, also nicht unerheblich finanzkräftiger war, als nach dem damaligen Finanzausgleich zu Grunde gelegt werden konnte.

32 Und auch die finanzschwachen Länder können nicht gegenüber ihren Bürgern in der Hoffnung großzügig sein, dass sie die minderen Steuereinnahmen durch einen Zuwachs im Finanzausgleich kompensieren können.

33 Bei dieser Reform haben vor allem die schwachen Länder sich dagegen gewehrt - während die starken geschwiegen haben -, die Gesetzgebungshoheit auch nur über die Steuern zu übernehmen, deren Ertrag ihnen ganz zukommt (s. näher Hans Meyer, Die Föderalismusreform 2006, 2008, S. 310 ff.). 
nivellierende Wirkung hat ${ }^{34}$ mit der Folge, dass die angenommenen Einnahmen mit den wirklichen Einnahmen pro Land nicht übereinstimmen (sollen). ${ }^{35}$

Von dem Grundsatz, dass alle Einnahmen der Länder einzustellen sind, hat das Finanzausgleichsgesetz 2001 eine bemerkenswerte Ausnahme gemacht. Nach § 7 Abs. 3 FAG sind nämlich die Einnahmen bei den Ländern geringer als tatsächlich aufgekommen anzusetzen, die im Vergleich zur Ländergesamtheit ein überdurchschnittliches oder beim allgemeinen Sinken der $»$ Veränderungsrate $\ll^{36}$ unterdurchschnittliches Ergebnis erzielt haben. Theoretisch kann das alle Länder treffen, wahrscheinlicher ist aber, dass die finanzstarken Länder eher Chancen zu einem überdurchschnittlichen Wachstum haben. Es handelt sich um einen Restbestand dessen, was unter dem Stichwort Wettbewerbsföderalismus ${ }^{37}$ damals energisch traktiert worden ist. Die Frage nach der Verfassungskonformität ist, soweit ersichtlich, nicht ernsthaft gestellt worden. Da man einig war und ohne Kläger nichts passieren konnte, und selbst wenn ein Land klagen würde, jedenfalls eine Reihe von Jahren bis zum Urteil gewonnen hätte, ist das auch nicht verwunderlich.

Wiederum ist die Begründung zum Gesetzentwurf aufschlussreich. Die Neuerung wird nicht an der Verfassung gemessen, sondern an $\S 3$ MaßstG. Sie sei, so die Begründung, »eine neue Komponente des bundesstaatlichen Finanzausgleichs «. ${ }^{38}$ Das kann man nicht bestreiten. $\S 3 \mathrm{MaßstG}$ deckt jedoch die Innovation nicht. Er formuliert ebenso luzide wie redundant: »Von Mehr- oder Mindereinnahmen gegenüber den länderdurchschnittlichen Einnahmen sowie von überdurchschnittlichen Mehreinnahmen oder unterdurchschnittlichen Mindereinnahmen je Einwohner gegenüber dem Vorjahr muss dem betreffenden Land ein Eigenbehalt bleiben. « ${ }^{39}$ Da der Finanzausgleich Summen nach ihrer Herkunft nicht identifiziert und es beim horizontalen nie zu

34 Die Wenigsten werden freilich auf Anhieb verstehen, wie das funktioniert, wenn sie den entsprechenden Gesetzestext, der von einer anzurechnenden »Steuerkraftzahl « spricht, lesen: »Als Steuerkraftzahlen werden für die einzelnen Länder die Beträge angesetzt, die sich ergeben, wenn die im Bundesgebiet insgesamt im Ausgleichsjahr aufgekommene Grunderwerbsteuer im Verhältnis der dem Aufkommen zu Grunde liegenden länderweisen Steuerbemessungsgrundlagen der Grunderwerbsteuer verteilt wird.« (\$ 7 Abs. 1 Satz 3 FAG).

35 Die nivellierende Wirkung bedeutet aber zugleich, dass es sich lohnt, die Steuermöglichkeiten auszunutzen, weil der über dem im Vergleich hergestellten Durchschnitt liegende Mehrertrag dem Land ohne Anlastungen aus dem Finanzausgleich verbleibt. Bei den Realsteuern, deren Ertrag den Gemeinden zukommt und für die sie das Hebesatzrecht haben, hat man denselben Mechanismus benutzt. Siehe dazu weiter Fn. 42.

36 Also prozentual mehr Steuereinnahmen oder weniger an Rückgängen als im Vorjahr hatten.

37 Die finanzstarken Länder, vor allem die Baden-Württemberger, weniger die Bayern, deren Vertreter Klaus Vogel sich zuletzt von dem baden-württembergischen Vorgehen distanziert hatte, und gar nicht die eher zum Prozess gezwungenen Hessen, hatten dies Mitte der 90er Jahre zur Grundlage ihres Generalangriffs auf den Finanzausgleich gemacht. Das Bundesverfassungsgericht hat dieses Vorbringen zwar zitiert (BVerfGE 101, 158, 198: »das dem Bundesstaatsprinzip zu entnehmende Gebot des föderalen Wettbewerbs«), es aber nicht einmal für erörterungswürdig befunden.

38 BT-Drucks. 14/7063 S. 26; auch für das Folgende.

39 Der ursprüngliche Gesetzentwurf kannte die Formel noch nicht (BT-Drucks.14/5951). 
einer vollen Abschöpfung kommen darf, ist dem Gebot immer Rechnung getragen. Erst recht verlangt $\S 3 \mathrm{MaßstG}$ nicht, dass die Finanzkraft nur verkürzt angesetzt wird. $\S 7$ Abs. 1 MaßstG verlangt dagegen, dass für den horizontalen Finanzausgleich grundsätzlich alle Einnahmen einzustellen sind. Um klarzustellen, was mit »grundsätzlich « gemeint ist, folgt eine Aufzählung »nicht ausgleichserheblicher « Einnahmen wie die Bagatellfälle oder die Äquivalente von Gegenleistungen der Landesseite oder gleichmäßig in allen Ländern anfallende und daher für den Ausgleich nicht erheblichen Einnahmen. Wenn man das schon nicht als eine abschließende Aufzählung begreifen will, so folgt doch daraus, dass die Einnahmen, die vom Ausgleich ausgenommen werden sollen, von Hause aus nicht »ausgleichserheblich« sein dürfen. Das ist bei dem hier angeordneten Eigenbehalt aber gerade der Fall. Es handelt sich um Einnahmen, die vom Typus her und auch nach der neuen Regelung in ihrem größten Umfang dem Ausgleich unterliegen, sie sind in dem betreffenden Land nur etwas besser angefallen als beim Durchschnitt der Länder. Die Begründung meint dagegen schlicht, die Aufzählung schließe »die Einbeziehung von Einnahmen in anderen begründeten Zusammenhängen nicht aus.« Die Aufzählung als solche möglicherweise nicht, wohl aber der Grundsatz der umfassenden Berücksichtigung aller Einnahmen, die als solche »ausgleichserheblich « sind.

Problematisch bleibt weiterhin die Berücksichtigung der Finanzkraft der Gemeinden. ${ }^{40}$ Auf dieses Gebiet hat sich offensichtlich 2001 die Auseinandersetzung zwischen den finanzstarken und den finanzschwachen Ländern konzentriert, und man ist zu einem von der Verfassung nicht gedeckten Kompromiss gelangt. Korrekt ist der Ausschluss der Einnahmen aus der Rechnung, welche die Gemeinden auf Grund des Art. 106 Abs. 7 GG, also im kommunalen Finanzausgleich, von den Ländern erhalten müssen oder können. Diese Gelder würden den Ländern sonst doppelt angerechnet, da es bei Art. 107 Abs. 2 Satz 1 GG allein um einen Vergleich der Länder geht. Korrekt ist auch der Versuch, neben den Anteilen an der Einkommen- und an der Umsatzsteuer, welche den Gemeinden nach Art. 106 Abs. 5 und 5a GG unmittelbar zukommen, auch die Einnahmen aus den Realsteuern (Art. 106 Abs. 1 Satz 1 Hs. 1 GG) zu erfassen. Da bei ihnen das Hebesatzrecht, also die Festlegung des Steuersatzes, den Gemeinden obliegt, wird wie bei der Grundstückserwerbsteuer, die vorstehend behandelt ist, eine normativ-nivellierende Berechnung vorgeschrieben, die aber die gesamten Einnahmen der Gemeinden in allen Ländern aus diesen Quellen erfasst ( 8 Abs. 2 FAG). ${ }^{41}$

Der Verfassung widerspricht dagegen die gänzliche Herausnahme der im Volumen nicht zu vernachlässigenden Konzessionsabgaben aus den kommunalen Einnahmen.

40 Obwohl das Grundgesetz in Art. 107 Abs. 2 GG auch die Finanzkraft der Gemeindeverbände anzurechnen befiehlt, geschieht das nicht, weil sich deren Einnahmen im Bagatellbereich bewegen.

41 Anders als bei der Grunderwerbsteuer besteht hier das Problem, dass keine landeseinheitlichen Steuerbemessungsgrundlagen existieren. Das Gesetz arbeitet mit Grundbeträgen, die sich aus der Gemeindefinanzstatistik des Statistischen Bundesamtes ergeben ( $\$ 8$ Abs. 2 FAG; die Erläuterung der Gesetzesbegründung dazu lässt sich als spartanisch bezeichnen, BT-Drucks. 14/7063 S. 27). 
Schon für das Jahr 1988 hatte die Bundesregierung gegenüber dem Bundesverfassungsgericht, das sich mit dem Thema intensiv auseinandergesetzt hat, ${ }^{42}$ die Konzessionsabgaben auf circa 4.1 Milliarden beziffert und erhebliche Schwankungen zwischen den Ländern konstatiert. ${ }^{43}$ Die Gesetzesbegründung konzediert, dass »sachliche Kriterien die Einbeziehung der Konzessionsabgaben nach einem normierten Soll-Aufkommen geboten erscheinen « lasse. ${ }^{44}$ Es existiert auch keine äquivalente und anrechenbare Belastung in den Gemeinden. Von der Konzessionsabgabenverordnung von $1992^{45}$, welche die Abgaben bundesweit einheitlich begrenzt, hatte das Gericht schon 1992 angenommen, sie werde »den Abbau der Verzerrungen $\aleph^{46}$ beschleunigen und angefügt: »Wenn dieser Abbau im wesentlichen eingetreten ist und die Konzessionsabgaben weiterhin in den einzelnen Ländern unterschiedlich anfallen, sind sie als Indikatoren gemeindlicher Finanzkraft in den Länderfinanzausgleich einzubeziehen. ${ }^{47}$ Diesen wenig interpretationsbedürftigen Befehl des Gerichts zitiert die Gesetzesbegründung vorsichtigerweise nicht. Sie behauptet auch nicht, dass die vor der Verordnung bestehenden Verzerrungen weiterhin bestünden; das wäre aber nach dem Urteil das einzige zulässige Argument der Nichtanrechnung. Dagegen wird eine Ermittlung des Soll-Aufkommens, wie es bei der Realsteuer praktiziert wird ${ }^{48}$ abgelehnt, weil es »äußerst fraglich« erscheine, ob die gegebenen Rahmenbedingungen der rechtlich autonomen Entscheidungen der Kommunen eine Ermittlung des Soll-Aufkommens $»$ vertretbar erscheinen lassen $\ll{ }^{49}$ Was immer mit den $»$ Rahmenbedingungen der rechtlich autonomen Entscheidungen der Kommunen « gemeint sein mag, ${ }^{50}$ einen solchen Vorbehalt bei der Ermittlung der Finanzkraft kennt weder die Verfassung noch die Rechtsprechung des Bundesverfassungsgerichts. ${ }^{51}$

In ihrer Wirkung zu Lasten der finanzschwachen Länder noch bedeutsamer als diese Einschränkung bei der Ermittlung der Finanzkraft der Gemeinden ist die pauschale Herabsetzung der so und also höchst defizitär ermittelten Gesamteinnahmen der Gemeinden auf lediglich 64\% ( $\$ 8$ Abs. 3 FAG). Das ist ein gewichtiger Vorteil für die finanzstarken Ländern, der sich ernsthaft nicht verteidigen lässt, und bei dem die Gesetzesmaterialien das in erstaunlicher Offenheit auch belegen.

42 BVerfGE 86, 148, $226 \mathrm{ff}$.

43 Siehe die Angaben in BVerfGE 86, 148, 226.

44 BT-Drucks. 14/7063 S. 27 1. Sp., wobei die Begründung ausdrücklich auf BVerfGE 86, 148,228 verweist.

45 BGBl. 1992 I 12.

46 Die wegen der meist langjährig geschlossenen Verträge bestanden.

47 BVerfGE 86, 148, 229.

48 Deren Steuersätze durchaus vergleichbare Spreizungen von Kommune zu Kommune aufweisen.

49 Drucks. 14/7063 S. 27 1. Sp. mit Verweis auf eine Expertise eines Wirtschaftsforschungsinstituts, das die Verfassung anders auslegt als das Bundesverfassungsgericht, sie aber nicht ändern kann.

50 Insbesondere, welcher Unterschied zu den Realsteuern besteht, die angerechnet werden.

51 Der Hinweis, dass es bei einer Anrechung des Ist-Aufkommens zu strategischem Verhalten von Kommunen zu Lasten der Zahlerländer kommen könnte, ist also irrelevant. 
Man könnte meinen, dieser Abschlag sei durch den ausdrücklichen Befehl der Verfassung in Art. 107 Abs. 2 Satz 1 GG gedeckt, den Finanzbedarf der Gemeinden nur $\mathrm{zu} »$ berücksichtigen«. Das Bundesverfassungsgericht hat sich sehr intensiv mit der Frage auseinandergesetzt und hat dabei auch eingehend untersucht, ob ein solches Vorgehen vielleicht durch diesen im Grundgesetz im übrigen unterschiedlich benutzten Begriff des »berücksichtigen« in Art. 107 Abs. 1 Satz 1 GG zu rechtfertigen sei. Es hat dies aus zwingenden Gründen in einem doppelten Sinne abgelehnt. Weder erlaube diese Formulierung, die Finanzkraft nur beschränkt anzurechnen, vielmehr sei sie grundsätzlich mit ihrem vollen Wert zu veranschlagen. Und zum anderen bedürfe auch der Abschlag durch Anrechnung eines außerordentlichen Bedarfs der Kommunen einer fundierten Analyse und Begründung. Beides ist einsichtig. Da die Finanzverfassung Land und Kommunen grundsätzlich als eine Einheit sieht (Art. 109 Abs. 9 GG), fehlt es schon daher an einem sinnvollen Grund, die Einnahmen beider unterschiedlich zu behandeln, und da es der Sinn des Ausgleichs ist, schwächere Länder zu Lasten der stärkeren besser zu stellen, ergäbe es keinen Sinn, schon bei den Ausgangszahlen der Rechnung Abschläge zuzulassen.

Oben ist schon auf die Merkwürdigkeit verwiesen, dass bei den Landeseinnahmen nicht ausdrücklich auch die Berücksichtigung der Landesbedarfe verlangt wird. Das war überflüssig, weil die Fixierung der Finanzkraft ohne eine Rückkoppelung an den Finanzbedarf nicht möglich gewesen wäre. Er ist durch die Kopfzahl dokumentiert. Dasselbe Element konnte aber als Äquivalent für die gemeindliche Finanzkraft nicht noch einmal genutzt werden, da es sich um dieselben Köpfe handelt. Der Finanzbedarf der Kommunen muss also, wenn er verfassungsrelevant sein soll, etwas davon, also vom allgemeinen Bedarf, Verschiedenes $\operatorname{sein}^{52}$ und er muss wie dieser so korrekt wie möglich erfasst werden.

Es liegt daher auf der Hand, dass der Ausführungsgesetzgeber zu Art. 107 Abs. 2 Satz 1 GG einen solchen über den allgemeinen Bedarf hinausgehenden Bedarf nicht einfach durch pauschale Abschläge von der Finanzkraft soll fingieren können, wenn denn die Verfassung ihre dirigierende Wirkung behalten soll.

Selbst die Gesetzesbegründung kann keinen einsichtigen Grund angeben, warum der Gesetzgeber einen so hohen Abschlag von den ermittelten Einnahmen der Kommunen toleriert hat. Im Gegenteil fehlt jegliche tragfähige Begründung. In seltener Klarheit ergeben die Gesetzesmaterialien, dass der Abschlag von über einem Drittel der Einnahmen ausschließlich auf einer Absprache zwischen dem Bundeskanzler nebst Bundesfinanzminister und den Ministerpräsidenten beruht, welche das Parlament zu exekutieren hatte und nur unter Knurren exekutiert hat.

Die Gesetzesbegründung liefert - ungewollt oder doch eher gewollt $-{ }^{53}$ hinreichende Gründe für diese Annahme. Wie es bei kritischen Gesetzesentwürfen, die unangenehme Vorgaben des Bundesverfassungsgerichts zu beachten haben, nicht unüb-

52 So auch BVerfGE 86, 148, 223.

53 Immerhin stammte der Entwurf aus dem Bundesfinanzministerium, das ein Interesse haben konnte, den möglichen Vorwurf verfassungswidrigen Verhaltens leicht weiterreichen zu können. 
lich ist, werden die Vorgaben penibel zitiert und dann ihre Einhaltung durch den Gesetzentwurf schlicht behauptet. So auch hier. Obwohl mehrmals betont wird, dass alle Einnahmen anzurechnen sind ${ }^{54}$ und obwohl das Gericht mit seiner Forderung zitiert wird, den »Finanzbedarf der Kommunen vorrangig an zuverlässigen Bedarfsindikatoren « zu bestimmen, und eingeholte Gutachten bestätigt haben, »dass der abstrakte Mehrbedarf der Gemeinden weitreichend anhand von Bedarfsindikatoren abgebildet werden kann «, ${ }^{55}$ haben sich Bundestag und Bundesrat nicht darum gekümmert, sondern damit begnügt, eine Einigung der Ministerpräsidenten mit dem Kanzler als ihre Entscheidung zu präsentieren, ${ }^{56}$ also eine Verhandlungslösung vorzuziehen, deren politische Rechtfertigung in der Einigung als solcher liegt, nicht aber in der Anwendung der Verfassung. Bei einem »weitreichend « objektiv feststellbaren abstrakten Mehrbedarf der Gemeinden kann der verbleibende Unsicherheitsfaktor nicht eine Absenkung der Anrechung der gemeindlichen Finanzkraft auf über ein Drittel rechtfertigen. Ehrlicher Weise versucht die Gesetzesbegründung auch nicht, den ja offensichtlich weitaus größeren Anteil objektiv feststellbarer - aber eben im Gesetzentwurf nicht festgestellten - Mehrbedarfe und die kleine Unsicherheitsmarge zu beziffern. Wenn beides zusammen über ein Drittel der Finanzkraft der Gemeinden ausgemacht hätte, wäre den so breit dargestellten und ausdrücklich wiederholten »hohen Begründungsanforderungen « des Bundesverfassungsgerichts im Gesetzentwurf Rechnung getragen worden. ${ }^{57}$ Dass es nicht geschah, kann nur den Grund darin haben, dass es nicht möglich war. Zusätzlich wird - eine weitere feine ministeriale Spitze - zum Schluss der Entwurfsbegründung zu $\S 8$ FAG auf die Bedeutung einer solchen »vollen Einbeziehung « als »Beitrag zur Annäherung der Finanzkraft der ostdeutschen Länder und ihrer Gemeinden an das Westniveau « hingewiesen, ${ }^{58}$ ein Anliegen, das der Entwurf gerade verfehlt. $^{59}$

54 BT-Drucks. 14/7063 S. 26 sub »Zu § 8 Steuereinnahmen der Gemeinden«, S. 27 1. Sp.

55 BT-Drucks. 14/7063 S. 28.

56 Die politische Technik besteht in solchen Fällen darin, Bundestag und Bundesrat vorweg über die erzielte Einigung der Ministerpräsidenten mit dem Kanzler positiv abstimmen zu lassen und dann die Ausführung in den Gesetzesgang zu geben. So ist es auch hier geschehen, wenn man die Gesetzesbegründung (BT-Drucks. 14/7063 S. 28) mit dem Verweis auf die jeweiligen Beschlüsse und die parlamentarische Debatte darüber kombiniert (s. z. B. BT-StenBer. 182. Sitzung, 17881f., 17884, 17893).

57 Um dem Letzten die Diskrepanz zwischen diesen Anforderungen und dem Verhalten des Gesetzgebers klar zu machen, schließt der Entwurf diese Passage mit dem Hinweis: »Dies hat sich der Gesetzgeber bei der Bemessung der Höhe des pauschalen Bedarfsabschlags zu vergegenwärtigen« (BT-Drucks. 14/7063 S. 28).

58 BT-Drucks. 14/7063 S. 28 zu $§ 8$.

59 Es mag sein, dass die ostdeutschen Ministerpräsidenten sich durch Versprechungen zum Solidarpakt II geschützt sahen, dadurch werden aber die verfassungsrechtlichen Anforderungen an das Finanzausgleichsgesetz nicht gemindert. Es enthält hinreichenden Spielraum für Verhandlungen, die auch vom Bundesverfassungsgericht toleriert werden, nicht aber bei der Festlegung der Ausgangsdaten. Die Stellung dieses Satzes in der Begründung deutet eher daraufhin, dass das Bundesfinanzministerium deutlich auf das Verfehlen dieses Anliegens hinweisen wollte. 
Dass der Bundestag bei diesem Verfahren zum bloßen Vollzugsorgan degradiert wird, kann vornehmlich die Opposition aussprechen. ${ }^{60}$ Die Regierungsfraktionen haben sich aber gehütet, ihr zu widersprechen. Die Fakten waren zu offensichtlich.

Die Behandlung der nach Art. 107 Abs. 2 Satz 2 GG grundsätzlich vollständig in Rechnung zu stellenden kommunalen Finanzkraft durch das Finanzausgleichsgesetz widerspricht also sowohl hinsichtlich der Nichtanrechnung der erheblichen Konzessionsabgaben als auch der Absenkung der Anrechnung der Steuerkraft auf nur 64\% der Verfassung. Immerhin machen allein die Steuereinnahmen der Gemeinden circa ein Drittel der eigentlichen Landeseinnahmen aus. Eine Klage dagegen dürfte sehr aussichtsreich sein, weil kaum zu erwarten ist, dass das Bundesverfassungsgericht seine Rechtsansicht in diesem Punkt ändert. Es handelt sich nicht um Felder, auf denen das politische Ermessen und eine Verhandlungslösung durch die Rechtsprechung zu tolerieren ist. Auch die Bundesregierung hatte in ihrer Gegenäußerung zur Stellungnahme des Bundesrates zum Entwurf des Maßstäbegesetzes unmissverständlich erklärt, dass sie die Abschläge bei den Einnahmen für unvereinbar mit der Rechtsprechung des Bundesverfassungsgerichts halte. ${ }^{61}$

Zudem würde eine vollständige Anrechnung es ermöglichen, die Tarife im Länderfinanzausgleich abzusenken, wie es nach dem Bericht des Sonderausschusses Maßstäbegesetz/Finanzausgleichsgesetz die Bundesregierung »klargestellt « hatte. ${ }^{62}$

Diese nicht unerhebliche Minimierung der Finanzkraft vor allem der finanzstarken Länder ${ }^{63}$ muss notwendig auf die Bewertung durchschlagen, ob der horizontale Finanzausgleich in Deutschland zu nivellierend wirkt. Dies wird auch jenseits der in diesem Punkt üblichen Propaganda ernsthaft debattiert, wobei vor allem finanzwissenschaftliche Überlegungen im Vordergrund stehen und manchmal den Anspruch eines normativen Datums erheben. Ein Nivellierungsverbot muss aber verfassungsrechtlich begründet werden, sollen aus ihm zwingende Folgerungen gezogen werden. Da es zudem auf Grundlagen unseres Verfassungs- und Finanzverfassungssystems verweist, soll ihm ein eigenes Kapitel gewidmet sein.

60 Siehe z. B. den Entschließungsantrag der FDP (»wieder einmal wurde eine wichtige politische Entscheidung außerhalb des Parlaments getroffen«: BT-Drucks. 14/6555) oder die Vorwürfe der FDP-Abg. Gisela Fricke, Mitglied und Mitberichterstatterin des entsprechenden Sonderausschusses, (»Wir hier im Parlament sind aber der eigentliche Gesetzgeber«, BT-Sten.Ber. 182. Sitzung, S. 17893), aber auch die Rede der Abgeordneten Antje Hermenau von BÜNDNIS 90/DIE GRÜNEN, die eingehend gerade auf die Behandlung der anzurechnenden Finanzstärke der Kommunen eingeht und zu berichten weiß, dass Franz Josef Strauß in der Zeit, als Bayern noch ein Nehmerland war, für die 100-prozentige Anrechnung der kommunalen Finanzkraft gefochten habe (a. a. O., S. 17881).

61 BT-Drucks. 1475971 S. 5.

62 BT-Drucks. 14/6533 S. 16 und S. 29.

63 Bei den Konzessionsabgaben wird man hier nach den in BVerfGE 86, 148, 226 berichteten Daten für die Stadtstaaten Einschränkungen machen müssen, für die kommunalen Steuereinnahmen gilt das nicht. 


\section{Die »erwirtschaftete Steuer« und das Nivellierungsverbot}

Das Grundgesetz verlangt in Art. 107 Abs. 1 Satz 1 GG zwingend einen angemessenen Ausgleich der unterschiedlichen Finanzkraft der Länder. Im Gegensatz dazu werden in Satz 3 Ergänzungszuweisungen des Bundes nur als möglich, also zulässig, bezeichnet. Grundsätzlich ist also der Ausgleich zwischen den Ländern vorzunehmen. In ihm verwirklicht sich »das bündische Prinzip des Einstehens füreinander «, das nach BVerfGE 72, 330, 386 »auch im Verhältnis der Länder untereinander gilt.« Dass der Ausgleich angemessen sein soll, ist zwar nicht sonderlich aussagekräftig, immerhin ist der Formulierung aber zu entnehmen, dass kein vollständiger Ausgleich erfolgen soll. Welchen Abstand er davon haben soll, lässt sich aus den Regeln der Finanzverfassung nicht entnehmen. ${ }^{64}$

Eine verbotene »Nivellierung der Länderfinanzen« taucht als Topos in einer sehr frühen Entscheidung des Bundesverfassungsgerichts auf, ${ }^{65}$ wird dort aber nur als ein möglicher Verstoß gegen »das bundesstaatliche Prinzip« erwähnt, ohne dass dies näher erörtert würde. Er taucht in den späteren Finanzausgleichs-Entscheidungen ${ }^{66}$ immer wieder auf, ${ }^{67}$ ohne dass aus ihm Folgerungen gezogen würden, die nicht schon aus dem Gleichbehandlungsgrundsatz ${ }^{68}$ entwickelt werden könnten, wie das Verbot, finanzstarke Länder zu finanzschwachen zu machen oder die Reihenfolge der Finanzstärke zu ändern. ${ }^{69}$ Die Angriffe gegen das Maß der Angleichung, welche das Finanzausgleichsgesetz in der jeweiligen Fassung vorgenommen hat, sind vor dem Bundesverfassungsgericht jedoch regelmäßig erfolglos gewesen.

Dagegen hat das Gericht mit seinen Argumentationen, vermutlich ohne es zu ahnen, vor allem in den jeweiligen Grundlegungen immer wieder denen Nahrung gegeben, die in dem unterschwellig erbitterten Streit ${ }^{70}$ um den Anteil an den öffentlichen Finanzen Chancen für einen erfolgreichen Angriff auf den bestehenden Finanzausgleich

64 Einen kleinen, aber nicht spezifischen Hinweis gibt Art. 107 Abs. 2 Satz 3 GG. Danach kann es trotz des Länderfinanzausgleichs noch so leistungsschwache Länder geben, dass der Bund einspringen darf. Daher muss der Ausgleich nicht so weit gehen, dass kein Land mehr relevant finanzschwach ist.

65 BVerfGE 1, 117, 131.

66 Die sich alle durch große Gesprächigkeit auszeichnen, was man wohl nur zum Teil als Reaktion auf die normalerweise außergewöhnlich umfangreichen Klageschriften und Äußerungen des Bundes und der anderen Regierungen erklären kann.

67 So in BVerfGE 72, 330, 398; 86, 148, 220, in der Sache auch S, 214f.; 101, 158, 222, 224 , 231f.; 116, 327, 380.

68 Siehe zum Beispiel BVerfGE 101, 158, 224.

69 Letzteres ist sowieso nur möglich, wenn die Finanzkraft eines Landes nicht ganz in die Ausgleichsrechnung eingestellt wird, wie das im FAG 1988 der Fall war. Deshalb sind die Formulierungen in BVerfGE 86, 148, 253 korrekt, in BVerfGE 116, 327, 381 dagegen nicht, wo von einer Anhebung der Finanzkraft eines Landes durch Bundesergänzungszuweisungen die Rede ist. »Finanzkraft « bezeichnet nämlich im Finanzausgleich die eigenen Einnahmen eines Landes, nicht Leistungen anderer Länder oder des Bundes an dieses Land.

70 So haben die finanzstarken Länder in einem Prozess einzelnen finanzschwachen Ländern die Existenzberechtigung abgesprochen (BVerfGE 101, 158, 199) und in demselben Prozess das damals »andersfarbige« Hessen, das zögerlich war, indirekt gezwungen, sich ihrer Klage anzuschließen. 
suchten. In diesem Zusammenhang sind die durchgängige Betonung der »staatlichen Eigenständigkeit der Länder «, des ihnen »Eigenen«, des »selbst Erwirtschafteten«, der »wirklichen Steuerkraft« nur einige Beispiele. Zusammen mit dem Modewort des Wettbewerbsföderalismus ergeben sie eine brisante politische Mischung.

Was zunächst das »selbst Erwirtschaftete $\ll^{71}$ angeht, so hört sich das zwar gut an wer gibt vom selbst Erwirtschafteten gerne etwas her -, ist aber nach der Verfassungslage nicht zum Nennwert zu nehmen. Nach Art. 105 GG ist der Bund der Herr der Steuergesetzgebung. Selbst im Bereich der konkurrierenden Steuergesetzgebung (Art. 105 Abs. 2 GG) ${ }^{72}$ haben bei der Föderalismusreform 2006 auch die starken Länder keinerlei Interesse gezeigt, die Gesetzgebungsverantwortung auch nur über diejenigen Steuern zu übernehmen, deren Ertrag ihnen allein zukommt. ${ }^{73}$ Warum sollten sie die politischen Kosten einer Steuererhöhung übernehmen, wenn ihnen der Bund das abnimmt. Den einzigen unmittelbaren und sehr aktiven Part beim »Erwirtschaften« eines Steuerertrages, den der Staat spielt, nämlich den Bürgern die Steuerlast aufzuerlegen, haben die Länder nicht übernehmen wollen. Dieser Teil des Erwirtschaftens obliegt also dem Bund und kann schwerlich ein Argument für die Länder abgeben. Die Steuerverwaltung gerade bei den beiden mit Abstand ertragreichsten Steuern ist zwischen dem Bund und den Ländern geteilt; zudem ist sie von Verfassungs wegen auf Gleichmäßigkeit der Besteuerung ${ }^{74}$ verpflichtet.

Wird denn die Steuer nicht wenigstens im jeweiligen Land erwirtschaftet? Der Frage hatte sich das Bundesverfassungsgericht angesichts der Regelungen des Zerlegungsgesetzes zu stellen. Art. 107 Abs. 1 Satz 2 GG sieht zwingend vor, dass durch Zustimmungsgesetz Bestimmungen vor allem über »Art und Umfang der Zerlegung des örtlichen Aufkommens « der Körperschaft- und der Lohnsteuer zu treffen sind. Bei der Körperschaftsteuer geht es um das Auseinanderfallen von Sitz der steuerpflichtigen Körperschaft und ihrer in anderen Ländern gelegenen Betriebsstätten ( $\$ 2$ Zerlegungsgesetz) und bei der Einkommensteuer um das Auseinanderfallen von Wohnort des Steuerpflichtigen und seiner in einem anderen Land belegenen Arbeitsstätte $(\S 7$ Zerlegungsgesetz). Diese hat die Lohnsteuer an das eigene Finanzamt abzuführen. Während bei der Körperschaftsteuer die im Sitzland der Körperschaft aufkommende Steuer (auch) auf alle anderen Länder mit eigenen Betriebstätten der Körperschaft aufgeteilt wird, hat das Sitzland der Arbeitsstätte des Lohnsteuerpflichtigen den Ertrag der Lohnsteuer an dessen Wohnsitzland zu überweisen, ein Problem vor allem für die Stadtstaaten mit ihren »Speckgürteln«. Während bei der Körperschaftsteuer das Zerle-

71 Siehe die Fundstellen in Fn. 6.

72 Die im übrigen durch die exzessive Rechtsprechung des Bundesverfassungsgerichts zu Art. 72 Abs. 2 GG (siehe dazu eingehend Hans Meyer, Föderalismusreform 2006, S. 101-102) in der Gefahr stehen, zu versteinern, da der Bund seine Kompetenz über »Modifizierungen « hinaus, nicht mehr ausüben kann.

73 Siehe Hans Meyer, a. a. O., S. $310 \mathrm{ff}$.

74 Im Verfassungsstreit der drei finanzstarken Länder Baden-Württemberg, Bayern und Hessen gegen den Finanzausgleich haben sie sogar vortragen lassen, dass sie die Lust »an der gleichmäßigen Durchsetzung der Besteuerung « verlieren könnten, wenn die Besserstellung der schwachen Länder zu weit getrieben werde (BVerfGE 101, 158, 199). Dass sie dasselbe auch für die schwachen Länder prophezeit haben, war wohl eher dem Anstand geschuldet. 
gungsgesetz also die Betriebsstätte maßgeblich sein lässt und nicht den Wohnort des Steuerpflichtigen, ist es bei der Lohnsteuer umgekehrt.

Es ist nicht so sehr interessant, dass das Bundesverfassungsgericht das toleriert hat (BVerfGE 72, 330, 406 ff. GG), sondern wie es argumentiert. Bei der Körperschaftsteuer blieben »die Steuern dort wo sie >erwirtschaftet ${ }^{75}$ worden sind «. Dass es bei der Lohnsteuer anders sei, wird mit dem zusätzlichen Verweis auf Art. 106 Abs. 5 Satz 1 GG »auch unter den Gegebenheiten eines einheitlichen Wirtschaftsraumes « lediglich als »nicht willkürlich« bezeichnet. Für den Nachsatz »in ihrer Verteilungswirkung erreicht sie eine erhebliche Annäherung an die wirkliche Steuerkraft der Länder « fehlt vorsichtiger Weise jede Begründung. Die Stadtstaaten werden zu Recht ein anderes Lied singen. ${ }^{76}$ Wenn BVerfGE 101, 158, 221 davon spricht, »Maßstab für diese ${ }^{77}$ horizontale Steuerertragsaufteilung ist das - im Zerlegungsgesetz verdeutlichte - »örtliche Aufkommen«, also das Entstehen von Steuerkraft im Zuständigkeitsbereich des jeweiligen Landes«, so kann es angesichts der Umsatzsteuerverteilung nach Köpfen nicht das »Erwirtschaften der Steuer « im Land meinen, sondern nur die Erhebung der Steuer im und für das Land.

Der Hinweis auf den »einheitlichen«, also nicht nach Landesgrenzen aufspaltbaren »Wirtschaftsraum ${ }^{78}$ führt zurück auf frühere Formulierungen, wonach Art. 107 GG dazu beitragen soll, eine Finanzordnung sicherzustellen, »die den Gesamtstaat und die Gliedstaaten am Ertrag der Volkswirtschaft sachgerecht beteiligt « ${ }^{79}$ oder in anderer Formulierung: »Darin kommt der von der Verfassung gefundene Kompromiss zum Ausdruck zwischen der auch auf finanzwirtschaftlichem Gebiet bestehenden Eigenstaatlichkeit der Länder und der sich aus dem Bundesstaatsprinzip ergebenden Gemeinschaft auch in der Teilhabe an der finanziellen Leistungsfähigkeit des Gesamtstaates. ${ }^{80}$ Bekanntlich gibt es keine bayerische oder mecklenburg-vorpommerische Volkswirtschaft und die Leistungsfähigkeit des Gesamtstaates ist etwas anders als die Leistungsfähigkeit der vereinigten Länder. Auch das lässt die Floskel, das jeweilige Land oder auch der Bund erwirtschafteten eine Steuer, in einem eher fahlen Licht erscheinen.

Wenn vielleicht auch nicht »erwirtschaftet«, so sind doch die Zahlungen im horizontalen Finanzausgleich aus dem »Eigenen« der Geberländer zu leisten. Das Bundesverfassungsgericht benutzt diesen Terminus häufiger und meint damit die Steuererträge, die den Ländern nach der Verfassung zukommen. ${ }^{81}$ Das wird auch als

75 Dass das Gericht den Terminus in Anführungszeichen setzt, zeigt eine gewisse und wohl auch berechtigte Unsicherheit.

76 Mit Sicherheit wird das für Berlin mit seinem hohen Anteil an öffentlicher Verwaltung nicht zutreffen.

77 Gemeint ist die durch Art. 107 Abs. 1 GG organisierte Verteilung.

78 BVerfGE 72, 330, 407.

79 So BVerfGE 72, 330, 388 mit Verweis auf BVerfGE. 55, 274, 300.

80 Nämlich nach der vollständigen Realisation des in Art. 107 Abs. 1 GG vorgesehenen Programms, BVerfGE 72, 330, 385f.

81 Z. B. BVerfGE 72, 330, 386, zwar sind die Finanzausgleichsleistungen rechtlich nicht aus den Steuereinnahmen zu bestreiten, faktisch aber sind diese die Grundlage. Mit Recht blendet das Bundesverfassungsgericht andere Einnahmen der Länder aus, seien sie im Finanzausgleich nicht anrechenbar, wie die Kredite, oder ohne relevante Bedeutung wie die Förderabgabe nach dem Bundesberggesetz. 
»originäre « Steuerausstattung bezeichnet. Das ist korrekt, weil sowohl die Ertragsverteilung der Einkommensteuer und der Landessteuern nach dem örtlichen Aufkommen als auch die Verteilung des Umsatzsteuerertrages nach der Kopfzahl oder höchstens eines Viertels davon primär nach Ausgleichsgesichtspunkten im Grundsatz verfassungsgeboten ist. Man muss dabei nur berücksichtigen, dass ein nicht unwesentlicher Teil dieser Erträge erst durch einfaches Gesetz endgültig festgelegt wird. Nämlich durch das Gesetz, das die Umsatzsteuererträge auf Bund und Ländergesamtheit aufteilt (Art. 106 Abs. 3 Satz 4 GG), das Gesetz, das vorweg den Gemeindeanteil bei der Einkommensteuer und der Umsatzsteuer festlegt (Art. 106 Abs. 3 Satz 1 GG), das Zerlegungsgesetz (Art. 107 Abs. 1 Satz 2 GG) und das Gesetz, das maximal ein Viertel des Umsatzsteuerertrages verteilt (Art. 107 Abs. 1 Satz 4 Hs. 2 GG).

Das »Eigene« steht also vielfältig zur Disposition des einfachen Gesetzgebers. Gleichwohl ist es die wichtigste finanzielle Basis der vom Gericht in diesem Zusammenhang immer wieder betonten Eigenstaatlichkeit der Länder. Da der Gesetzgebungsherr sowohl über die Steuern selbst wie über die Verteilung eines nicht unwesentlichen Teils der Erträge der Bund ist, fehlt der Eigenstaatlichkeit der Länder nach unserer Finanzverfassung aber ein wichtiger Bestandteil, nämlich selbst über die eigenen Steuereinnahmen zu disponieren. Mit der »auf finanzwirtschaftlichem Gebiet bestehenden Eigenstaatlichkeit ${ }^{82}$ ist es also in einem wichtigem Punkt nicht weit her.

Für die finanzschwachen Länder sind die nach Art. 107 Abs. 2 Satz 1 GG notwendigen, und ebenfalls durch Bundesgesetz zu organisierenden Ausgleichleistungen ebenfalls Bestandteil ihrer finanziellen Ausstattung und in gleicher Weise wie die originären Steuererträge Basis der eigenstaatlichen Aktivitäten. Die Verfassung kennt insofern keinen Unterschied und die gelegentlich auch in den Urteilen durchscheinende Vorstellung unterschiedlicher Wertigkeit der Herkunft der Finanzmittel ${ }^{83}$ findet in ihr jedenfalls keine Stütze.

Es bleibt der Ruf nach dem »Wettbewerbsföderalismus«. Er ist selbstverständlich kein Gedanke der Verfassung und das Bundesverfassungsgericht hat die vor allem von Baden-Württemberg vorgetragene Idee eines «in Art. 20 Abs. 1 und Abs. 2 GG abgesicherten Prinzips des föderalen Wettbewerbs $«{ }^{84}$ in der Begründung seines Urteils nicht einmal der Erwähnung für wert befunden. Der Gedanke des Wettbewerbs passt nicht, weil die Länder die dafür notwendige Freiheit zum Egoismus nicht besitzen, sondern vielfältig gebunden sind und weil es keinen Markt gibt. Wenn es einen Markt gäbe, hätten die Bayern, die jahrzehntelang vom Finanzausgleich profitierten, SPD wählen müssen, weil die Gelder zu einem nicht unerheblichen Teil vom damals SPD-regierten Hessen kamen. Sie haben das selbstverständlich nicht getan. Andererseits hat der immer

82 Siehe vorstehende Fundstelle.

83 In BVerfGE 116, 327, 379 wird die »eigene Finanzausstattung der einzelnen Länder« auf die Zuflüsse allein aus Art. 107 Abs. 1 GG beschränkt, zugleich aber mit Verweis auf Stefan Korioth (Der Finanzausgleich zwischen Bund und Ländern, 1997, S. 421 f.) angedeutet, dass es Kritik an dieser Feststellung gebe, was nur als Vorbehalt späteren Umdenkens gedeutet werden kann. Warum hätte das Gericht sonst auf sie verwiesen? Denn auf die Kritik eingelassen hat sich das Gericht nicht.

84 BVerfGE 101, 158, 198f. 
wieder beschworene mangelnde Anreiz unseres Finanzausgleichs die Bayern nicht gehindert, in die Position eines Nehmerlandes zu wechseln. Es ist sicher richtig, dass nicht der Finanzausgleich ein Nehmerland dazu bringt, Gewerbe- und Industrieansiedlung zu betreiben, die nächste Wahl lässt sich aber in einem stagnierenden Land schlecht gewinnen. Eine Rikscha-Mentalität ist unabhängig von jedwedem Finanzausgleich politisch unklug und daher mehr als unwahrscheinlich. Es geht immer um die Wiederwahl.

Es darf auch daran erinnert werden, dass beim gesamten horizontalen Finanzausgleich die Verschuldung eines Landes keine Rolle spielt. Es geht nur um die ProKopf-Ressourcen eines Landes.

Als reines politisches Spielgeld darf die Argumentation gewertet werden, ohne die Last aus dem horizontalen Finanzausgleich hätten sich die finanzstarken Länder nicht oder nicht so verschuldet. Seit der Finanzverfassungsreform 1969, als die Politik entgegen dem Vorschlag der Troeger-Kommission die Schleusen der Verschuldung geöffnet hat, ${ }^{85}$ gehört das Leben über die eigenen Verhältnisse zum Alltag der Politik. Man liegt nicht weit von der Wahrheit, wenn man die Verschuldung auch als das Doping der Politik bezeichnet; es ist wie im Sport schwer zu bekämpfen. Nichts spricht dafür, dass die finanzstarken Länder, die eine Verschuldung nicht oder nicht in dem getätigten Maße nötig gehabt haben, bei geringerem Einzahlen in den horizontalen Finanzausgleich auf einmal clean geworden wären.

\section{Die Bundesergänzungszuweisungen}

Kein Teil der Finanzverfassung leidet so unter terminologischer Verwirrung und damit auch Missverständnissen wie das Institut der Bundesergänzungszuweisungen. Richtig ist zunächst die Feststellung des Bundesverfassungsgerichts, dass die Bundesergänzungszuweisungen die vierte, nicht notwendig obligatorische ${ }^{86}$ Stufe der Finanzverteilung im Bundesstaat darstellt. ${ }^{87}$ Mit seiner Regelung, dass der Bund »leistungsschwachen Ländern zur Deckung ihres allgemeinen Finanzbedarfs (Ergänzungszuweisungen)« gewähren kann, bringt Art. 107 Abs. 2 Satz 3 GG zwei neue Begriffe in die Terminologie des Finanzausgleichs ein, nämlich den der leistungsschwachen Länder und den des allgemeinen Finanzbedarfs (der Länder). Ersteres hat BVerfGE 72, 330, 402 zu der naheliegenden Behauptung verleitet, die Finanzschwäche sei nicht aufkommensorientiert wie der Begriff der Finanzkraft in Art. 107 Abs. 2 Satz 1 GG, sondern bezeichne »eine Relation zwischen Finanzaufkommen und Ausgabenlasten der Länder «. Es hat dabei aber die eigene Einsicht vergessen, dass der Begriff »Finanzkraft« in Art. 107 Abs. 1 Satz 1 GG sich ohne Bezug zu einer Ausgabenlast nicht sinnvoll definieren lässt, ${ }^{88}$ also eine

85 Siehe näher Hans Meyer, Solidität und Verantwortung. Einige Bemerkungen zur Staatsverschuldung in: Föderalismuskommission II: Neuordnung von Autonomie und Verantwortung (Hrsg. Kai A. Konrad / Beate Jochimsen), 2008, S. 91 ff.

86 Das Gericht hat angedeutet, dass sich das Können unter bestimmten Umständen auch zu einer Pflicht verdichten kann. Sehr großzügig in BVerfGE 72, 330, 403, freilich mit der eigenartigen Einschränkung, dass die Leistung »praktisch « zur Pflicht werde.

87 Exemplarisch in BVerfGE 72, 330, 383 ff., ähnlich in BVerfGE 86, 158, $219 \mathrm{ff}$.

88 BVerfGE 72, 330, 400, aufgenommen in BVerfGE 86, 148, 223. 
entsprechende Relation darstellt. Es hat bei der Fixierung der Finanzkraft nach Art. 107 Abs. 2 Satz 1 GG nämlich lediglich »besondere Aufgabenlasten« der Länder ausgeschlossen und die normale Aufgabenlast durch die »Kopf-Wertung « als sinnvoll abgedeckt angesehen ${ }^{89}$ In beiden Fällen geht es also entgegen - und eigenartigerweise mit dem Gericht um Relationen zwischen Finanzstärke und Aufgabenlast. Richtig ist nur, dass dasselbe Problem einmal aus der Sicht der Finanzstärke und das andere Mal aus der Sicht der Finanzschwäche angegangen wird.

Das hat auch Konsequenzen, weil das Gericht aus seiner Sicht schließt, dass der Bund auch »Sonderlasten « einzelner Länder berücksichtigen darf ${ }^{90}$ und daraus wiederum folgert, dass das Volumen der Ergänzungszuweisungen des Bundes die des horizontalen Finanzausgleichs beträchtlich übersteigen kann. ${ }^{91}$ Das dürfte als allgemeine Aussage dem Verhältnis von obligatorischem Länderfinanzausgleich und fakultativer vierter Stufe der Finanzverteilung wohl kaum gerecht werden. Schon in BVerfGE 86, 148 ist die These denn auch stillschweigend korrigiert worden. ${ }^{92}$

Die zweite terminologische Verwirrung gibt es um den Begriff der Finanzkraft. Er meint dort, wo er von der Verfassung benutzt wird, nämlich in Art. 107 Abs. 1 Satz 1 GG, auch nach dem Bundesverfassungsgericht die Einnahmepotenz des Landes, die sich vornehmlich, aber nicht unbedingt ausschließlich, in seiner Steuerkraft ausdrückt. Er meint nicht den Anteil an Finanzpotenz des Landes, die sich zusätzlich aus möglichen Ausgleichsleistungen im horizontalen Finanzausgleich speist. In BVerfGE 72, 330, 404 umfasst der Begriff »Finanzkraft« neben den erhaltenen Leistungen im horizontalen Finanzausgleich aber auch mögliche Ergänzungszuweisungen selbst. Das ist nun mehr als verwirrend. ${ }^{93}$

Die sauberste Lösung wäre, es bei der Definition der Finanzkraft in Art. 107 Abs. 1 Satz 1 GG zu belassen, welche die Steuerkraft eines Landes nach Art. 107 Abs. 1 GG sowie mögliche andere anrechnungsfähige Einnahmequellen wie die Bergabgabe erfasst und die in Relation zur Kopfzahl des Landes gesetzt wird. Zusammen mit möglichen Einnahmen aus dem horizontalen Finanzausgleich ergibt das die Finanzstärke (nicht: Finanzkraft) eines Landes. Ist diese unterdurchschnittlich, handelt es sich um ein »finanzschwaches« Land im Sinne des Art. 107 Abs. 2 Satz 3 GG.

89 Sowohl in BVerfGE 72, 330, 401 als auch in BVerfGE 86, 149, 223, wobei dies als ein »abstrakter« Bedarf bezeichnet ist und zugleich strukturell begründete Veredelungen der Köpfe zugelassen worden sind. Siehe dazu oben S. 128, Fn. 29.

90 BVerfGE 72, 330, 402; auch für das Folgende.

91 So muss man wohl die Formulierung verstehen, dass sie »im Verhältnis zum horizontalen Finanzausgleich ein beträchtliches Volumen erreichen« können.

92 Jedenfalls bei Berücksichtigung von »Sonderlasten aus einer Haushaltsnotlage« könne das »nur in dem Umfang geschehen, der der Funktion der Bundesergänzungszuweisungen im Rahmen des Finanzausgleichs als dessen letztere Stufe entspricht.« Restriktiv auch BVerfGE 101, 158, 233.

93 Es hat seine Parallele im Weiterschleppen des Verbots der Veränderung der Finanzkraftreihenfolge, das nur einen Sinn ergibt, wenn die Finanzausgleichsregeln von vorne herein Finanzkraft im Sinne des Art. 107 Abs. 1 GG nicht in die Ausgleichsrechnung einstellt (s. dazu Fn. 68). 
Kein Augenmerk widmet das Gericht in seiner ersten Grundentscheidung zum Thema dem Merkmal, dass die Ergänzungszuweisung an Länder zur »ergänzenden Deckung ihres allgemeinen Finanzbedarfs« zu gewähren ist. Das hätte um so eher nahegelegen, als das Gericht sich vorrangig mit der Berücksichtigung von Sonderlasten eines Landes befasst. In der bisher letzten Entscheidung zum Thema, dem Berliner Fall, wird das Gericht sensibler und merkt, dass die Sonderlastentheorie einer Eingrenzung bedarf, die sich nicht nur, aber vor allem auf die Sonderlast eines Haushaltsnotstandes bezieht. ${ }^{94}$

Eine systematischere Betrachtung würde zunächst zurückgehen auf die Philosophie des horizontalen Finanzausgleichs, deren Ergänzung nach der ständigen Rechtsprechung $^{95}$ nur die Ergänzungszuweisungen sein dürfen. Da die dort genannte »Finanzkraft« die Einnahmepotenz des Landes im Verhältnis zu seiner Einwohnerzahl meint, geht die Verfassung offenbar davon aus, dass das Land mit den entsprechenden, vor allem aus den Steuererträgen stammenden Mitteln seine Aufgaben von der Sicherheit über die Infrastruktur bis zur Bildung und Kultur, Wirtschaftsförderung usw. soll erledigen können. Das bedeutet auch die Verpflichtung, das Maß und die Opulenz seiner Aufgabenerfüllung danach auszurichten. Da die Steuerkraft nach dem örtlichen Aufkommen aber unterschiedlich ist und die Ergänzungsanteile an der Umsatzsteuer nach Art. 107 Abs. 1 Satz 4 Hs. 2 GG die Differenz nicht immer ausgleichen können, verlangt Art. 107 Abs. 2 Satz 1 GG einen horizontalen Ausgleich zwischen den finanzkraftstarken und den finanzkraftschwachen Ländern, der aber nicht zu einer Nivellierung der Unterschiede führen darf. Darum kann aber weiterhin eine untragbare Differenz zwischen Ressourcen und auch sparsamer Aufgabenerfüllung bestehen bleiben. Diese kann der Bund mildern, und zwar durch Ergänzungszuweisungen zur Deckung des, wie Art. 107 Abs. 2 Satz 3 GG ausdrücklich bestimmt, nur »allgemeinen« Finanzbedarfs.

Nimmt man das ernst, dann ist die Variante des Gerichts, es seien auch Sonderbedarfs-Ergänzungszuweisungen zulässig, zumindest fragwürdig. Auch hier lässt sich der Grund in der Ausgestaltung des horizontalen Finanzausgleichs finden. Er hat mit dem Pro-Kopf-Ansatz einen sehr allgemeinen, aber auch fundamentalen Bedarfsmaßstab. Mit der Erlaubnis zur »Veredelung « der Köpfe reagiert dieser Ausgleich auf strukturelle Mehrbedarfe wegen der Bevölkerungsdichte ${ }^{96}$ bei den Stadtstaaten und wegen sehr geringer Bevölkerungsdichte bei einigen Flächenländern. Nun kann es aber auch andere strukturelle Mehrbedarfe geben, die über die Pro-Kopf-Verteilung nicht eingefangen werden können. Ein Beispiel ist der Infrastruktur-Nachholbedarf der Ostländer und die unterdurchschnittliche Finanzkraft ihrer Gemeinden, die in $\S 11$ Abs. 2 FAG durch Bundesergänzungszuweisungen aufgefangen werden. Da es sich um einen ebenso allgemeinen Mehrbedarf handelt wie bei den höheren Kosten eines dünnen Besiedlung, ist die Bezeichnung der entsprechenden Bundesleistung als Son-

94 BVerfGE 116, 327, 382.

95 Zuletzt BVerfGE 116, 327, 381.

96 Wie oben gezeigt, ist das Vorliegen solcher Mehrbedarfe nicht unstrittig, jedenfalls aber nicht widerlegt. 
derbedarfs-Bundesergänzungszuweisung irreführend. Es handelt sich um einen Normalfall des Art. 107 Abs. 2 Satz 3 GG. Der Unterschied besteht lediglich darin, dass der Mehrbedarf kein dauernder ist, weil die Hilfe gerade zu seiner Beseitigung geleistet wird. Daher ist die nicht unerhebliche Absenkung von 10 auf 2 Milliarden im Jahre 2019 konsequent.

Auch die strukturelle Arbeitslosigkeit in den Ostländern erzeugt einen strukturellen Mehrbedarf und keinen Sonderbedarf. Er wird durch die Pro-Kopf-Verteilung nicht erfasst, ist aber ein markantes Belastungsmoment für die Länder. Es handelt sich also ebenfalls trotz ihrer entsprechenden Kennzeichnung nicht um eine Sonderbedarfs-Ergänzungszuweisung. § 11 Abs. 3a FAG sieht keine Absenkung der Bundesleistung von einer Milliarde vor. Das ist vertretbar, weil eine Absenkung zwar eine mögliche Nebenfolge, nicht aber das Ziel der Leistung ist. Korrekt ist aber die vorgesehene Überprüfung im Jahre 2010, die sowohl die Notwendigkeit wie die Höhe der Leistung betrifft.

Wenig einsichtig ist dagegen die Anerkennung »überdurchschnittlich hoher Kosten der politischen Führung « als »Sonderbedarf« (§ 11 Abs. 4 FAG). Der Bund wendet dafür eine halbe Milliarde auf und bedenkt damit die fünf neuen Bundesländer sowie fünf finanzschwache alte Bundesländer von Berlin über Bremen, Saarland, RheinlandPfalz bis Schleswig-Holstein. ${ }^{97}$ Es handelt sich nicht um einen strukturellen Mehrbedarf. Ein finanzschwaches Land hat sich in der Organisation und Ausstattung seiner politischen Führung, was immer man darunter zu verstehen hat, seinen finanziellen Möglichkeiten anzupassen. Das geschieht in anderen Bereichen des Landes auch. Es handelt sich um einen Bedarf, der im horizontalen Finanzausgleich hinreichend bedient ist. Außerdem erhalten die finanzschwachen Länder, die im horizontalen Finanzausgleich eine in $\S 11$ Abs. 2 FAG näher beschriebene Annäherung an einen Durchschnitt nicht erreichen, allgemeine Bundesergänzungszuweisungen für ihre allgemeinen Aufgaben, wozu selbstverständlich auch die politische Führung eines Landes gehört. Die in $\S 11$ Abs. 4 FAG vorgesehenen Zuweisungen des Bundes entsprechen nicht der Verfassung.

Ein Antrag Berlins auf Bundesergänzungszuweisungen zur Haushaltssanierung hat zu einer ablehnenden, auch über den Fall hinaus außerordentlich restriktiven und bisher letzten Entscheidung des Bundesverfassungsgerichts zur Finanzverfassung geführt. ${ }^{98}$ Großzügigere Äußerungen vorangegangener Urteile wurden dabei zu Recht revidiert. Auch hier lohnt es sich, einen Blick auf die Philosophie des Finanzausgleichs zu werfen. Er ist - nicht nur nach ständiger Rechtsprechung - dazu da, Bund und Länder zur Erfüllung ihrer Aufgaben hinreichend auszustatten. Dabei wird der jeweilige Bedarf und werden auch strukturelle Mehrbedarfe, wie gezeigt, in Rechnung gestellt. Die Verschuldung fehlt aus gutem Grund in dieser Rechnung. Die Glieder des Bundesstaats haben sich bei ihrer Ausgabengestaltung an die durch Steuerertrag, Finanzausgleich und sonstige Einnahmen gegebenen Möglichkeiten zu halten. Nehmen

97 Zehn Länder mit durchschnittlich 50 Millionen zu bedenken, ist natürlich bei den unumgänglichen Verhandlungen ein schönes Spielgeld des Bundes.

98 BVerfGE 116, $327 \mathrm{ff}$. 
sie Schulden zur Ausweitung ihrer Handlungsmöglichkeiten auf, tun sie das auf eigenes Risiko. Jedwede Anerkennung der Schulden als ein im Finanzausgleich zu berücksichtigender Bedarf, würde dieses Risiko zu einem nicht unwesentlichen Teil auf die anderen Glieder des Bundesstaates verlagern, was der bündischen Solidarität widerspräche.

Das Problem der eingerissenen Verschuldung der öffentlichen Hand besteht darin, dass sie die aufgenommenen Kredite nicht zurückzahlt, was nicht nur dem Sinn eines jeden Kredits widerspricht, sondern auch den meist vorhandenen Restriktionen in den Verfassungen für die Kreditaufnahme. Die Kredite werden vielmehr dauernd revolviert und so gestapelt. ${ }^{99}$ Als Belastung erscheinen nur noch die Zinszahlungen, ${ }^{100}$ die zum Beispiel beim Bund im Jahr höher sind als die von ihm getätigten Investitionen. Diese absurde Situation führt dazu, das die Verschuldung als Puffer bei unerwarteten Ausgabenpflichten, sei es bei Naturkatastrophen oder einem scharfen Einbruch der Konjunktur, ausfällt. Diesen Spielraum müssen aber die Länder (und auch der Bund) behalten, wenn sie eine eigenverantwortliche Politik betreiben wollen.

$\mathrm{Zu}$ Recht hat das Bundesverfassungsgericht judiziert, dass das keine Probleme des Finanzausgleichs sind: »Bundesergänzungszuweisungen dienen bei alledem nicht dazu, augenblicksbedingte finanzielle Notstände zu beheben, aktuelle Projekte zu finanzieren oder finanziellen Schwächen abzuhelfen, die eine unmittelbare und vorhersehbare Folge von politischen Entscheidungen eines Landes bilden« (BVerfGE 116, 327, 382).

Wenn man dem Gericht folgt - und es spricht viel dafür -, dann bleiben Sonderbedarfs-Bundesergänzungszuweisungen nur für die Ultima-ratio-Fälle, also vermutlich für die Fälle, in denen sowieso nicht vorrangig nach der Verfassung gehandelt werden wird.

99 Siehe näher Hans Meyer, a. a. O. (s. Fn. 86), S. 96 ff.

100 Selbst das Bundesverfassungsgericht scheint vergessen zu haben, dass die Rückzahlungsverpflichtung auch zu den Lasten eines Kredits gehört, wenn es nur von »übermäßigen Belastungen durch die Rechtspflicht zu Zinszahlungen« spricht (BVerfGE 116, 327, 385). 